\title{
The Best Laid Plans—Medication Reconciliation Optimization in Theory and Practice
}

\author{
Vicki I-Sing Jue, PharmD, BCGP1*, Raman Ravi Khanna, MD, MAS²
}

'Pharmaceutical Department, University of California, San Francisco, San Francisco, California; ${ }^{2}$ Division of Hospital Medicine, University of California, San Francisco, San Francisco, California.

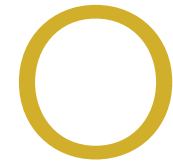

$f$ all the errors that occur in modern healthcare, medication errors are among the most ubiquitous and consequential. Adverse drug events (ADEs) account for approximately 700,000 emergency department visits, 100,000 hospitalizations, and 1.3 million people are injured by medication errors annually. ${ }^{1}$ Among the most frequent causes of preventable ADEs are errors on the medication lists when patients are admitted to hospitals. ${ }^{2}$ Therefore, preventing discrepancies between medications the patient is prescribed (and actually taking) inside and outside the hospital-the so-called "medication reconciliation"-is an intense, ongoing area of focus for health systems, pharmacies, and numerous quality and safety organizations seeking to reduce ADEs.

Past studies of medication reconciliation interventions have suggested benefit from restricting medication reconciliation to admission or discharge, pharmacist or pharmacy technician-led medication reconciliation, and pharmacy-led interventions (ie, telephone follow-up/home visit, patient counseling) for ensuring an accurate medication list. ${ }^{3-5}$ Recent evidence suggests that pharmacist discharge medication reconciliation is associated with decreased readmission rates, decreased medication discrepancies, and adverse events associated with drug therapy issues. ${ }^{4}$ The successful interventions were promising, but disseminating such interventions can often be very complex. ${ }^{6}$

In this issue of the Journal of Hospital Medicine, Mixon et al. report the results of a subanalysis of the MARQUIS trial, wherein they individually examined the on-protocol effects of the interventions that MARQUIS recommended, comparing hospitals to their own running baseline data at the implementation of each intervention to data following the implementation. The authors found that only three of the nine interventions were associated with reducing potentially harmful discrepancies in the medication list-training existing staff to perform discharge medication reconciliation, hiring additional staff for this purpose, and defining roles and responsibilities and roles clearly - and that two were actually associated with harm - training existing staff to take best possible medication histories (BPMHs) and implementing a new Electronic Medical Record (EMR). MARQUIS is unique in not just attempting

*Corresponding Author: Vicki Jue, NCGP; E-mail: I-SingVicki.Jue@ucsf.edu; Telephone: 415-353-1095

Received: June 27, 2019; Accepted: June 28, 2019

๑ 2019 Society of Hospital Medicine DOI 10.12788/jhm.3281 but in reporting "best case" real-world implementation using available literature to design mentored, practical approaches to those same interventions at sites not involved in their initial setup and validation.

EMR implementation should in theory improve accuracy (or at least legibility), but it can also contribute to new types of inaccuracy or, as the authors propose, deprioritize quality and safety as organizational goals during the rigors of digitization. Similarly, training staff to take a BPMH might create false confidence in the results or interact with medication reconciliation in other complex ways. Opting to add more work instead of hiring additional staff may have increased the burden of medication review and thus contributed to its inaccuracy.

On the contrary, certain interventions, such as having clear accountability for the medication list, hiring additional staff to construct that list, and clearly defining the roles of those involved in the reconciliation process, were associated with improved medication reconciliation. All these strategies require resource allocation, but at least the current study provides evidence that such resource allocation can be effective in new settings as they were in their original ones.

The study has important acknowledged limitations. The on-protocol analysis limited the authors to reporting associations rather than causality. Moreover, the original trial ran from 2011 to 2014, which was a time of rapid EMR implementation and new recognition of the problems posed by the same; several organizations are in a far more mature EMR context today. Conversely, newer technologies such as patient-facing medication reconciliation applications, cross-organization medication lists available from some EMR vendors, and health platforms that collect data from multiple EMRs were not evaluated because they did not exist at the time of the original trial. Another important trend in healthcare, the rise of Accountable Care Organizations and their focus on integration and defragmentation, may have an important part to play in medication list accuracy. All the above-mentioned aspects will be important avenues for ongoing research in real-world medication reconciliation.

Mixon's findings come at a time when medication reconciliation is again a national health informatics priority, a key component of the Medicare Access and CHIP reauthorization Act of 2015 and Merit-based Incentive Payments System ${ }^{8}$ since 2019, with hospitals reporting medication reconciliation rates for financial in addition to quality and safety reasons. Hopefully, this study and others, in combination with the abovementioned incentives, will stimulate further research into impactful strate- 
gies for medication reconciliation and ideal ways to implement them. With luck, the end result will be more generalizable interventions, with a track record of success, that would help ensure that patients are prescribed, are reporting, are taking, and are noted to be taking the medications that they and their providers intended, both on presentation to the hospital and on discharge home.

Disclosures: Vicki Jue has no conflicts of interest to report. Raman Khanna reports developing CareWeb, a communication platform that has been licensed to Voalte, Inc. This work is unrelated to the current editorial. No other conflicts of interest to report.

\section{References}

1. Center for Drug Evaluation and Research. Medication Errors-Medication Error Reports. https://www.fda.gov/Drugs/DrugSafety/MedicationErrors/ ucm080629.htm.Accessed June 14, 2019.
2. Cornish PL, Knowles SR, Marchesano R, et al. Unintended medication discrepancies at the time of hospital admission. Arch Intern Med. 2005;165(4):424429. https://doi.org/ 10.1001/archinte.165.4.424.

3. Mekonnen AB, McLachlan AJ, Brien JE. Pharmacy-led medication reconciliation programmes at hospital transitions: a systematic review and meta-analysis. J Clin Pharm Ther. 2016;41(2):128-144. https://doi.org/ 10.1111/ jcpt.12364.

4. Kilcup M, Schultz D, Carlson J, et al. Postdischarge pharmacist medication reconciliation: impact on readmission rates and financial savings. J Am Pharm Assoc. 2013;53(1):78-84. https://doi.org/ 10.1331/JAPhA.2013.11250.

5. Cater SW, Luzum M, Serra AE, et al. A prospective cohort study of medication reconciliation using pharmacy technicians in the emergency department to reduce medication errors among patients. J Emerg Med. 2015;48(2):230238. https://doi.org/ 10.1016/j.jemermed.2014.09.065.

6. Horton TJ, Illingworth JH, Warburton WHP. Overcoming challenges in codifying and replicating complex health care interventions. Health Aff. 2018;37(2):191-197. https://doi.org/ 10.1377/hlthaff.2017.1161.

7. Mixon A, Kripalani S, Stein J, et al. An on-treatment analysis of the MARQUIS study: interventions to improve inpatient medication reconciliation. J Hosp Med. 2019;(10):614-617. https://doi.org/ 10.12788/jhm.3258

8. Centers for Medicare \& Medicaid Services. https://www.cms.gov/Medicare/ Quality-Initiatives-Patient-Assessment-Instruments/Value-Based-Programs/MACRA-MIPS-and-APMs/MACRA-MIPS-and-APMs.html.Accessed June 25, 2019. 